Rev. Adv. Mater. Sci. 56 (2018) 42-53

\title{
THERMODYNAMICS OF NATURAL PIGMENT-DOPED ETHANOL UNDER MONOCHROMATIC LASER IRRADIATION
}

\author{
Miguel de Icaza Herrera and Víctor M. Castaño \\ ${ }^{1}$ Centro de Física Aplicada y Tecnología Avanzada, Universidad Nacional Autónoma de México, A.P. 1-1010, \\ Querétaro, Qro 76000, México
}

Received: January 19, 2018

\begin{abstract}
A laser beam heats a mixture of ethanol and anthocyanins producing the effect of a reciprocating machine. This behaviour is analyzed in terms of the stable, metastable and unstable states of Landau and Lifschitz. Trying first with the van der Waals equation of state, with negative results because the system state is normally far from the critical state, a modified version, with van der Waals coefficients depending linearly on the temperature is proposed, based in the thermodynamic properties of ethanol at room and critical temperatures. The resulting theory allows us to understand the discontinuous results concerning the absorption of the laser beam in terms of a thermodynamic cycle and its frequency dependence on the laser light focus position. Slight changes in the experimental setup may enable the measurement of thermodynamic properties in metastable states.
\end{abstract}

\section{INTRODUCTION}

The relationship between optical and physicochemical properties of natural pigments have attracted increased attention in the literature since Graetzel's pioneering work [1] not only for the interesting basic science involved, but also because of their use for solar cells and other optoelectronic devices. Indeed, a number of natural produces, such as betalains [2,3], carotenoids [4,5], chlorophyll [5], bixin [6] and many others, have been studied in terms of both their chemical behavior and stability under light, aiming to produce novel, environmentallyfriendly and durable components for energy harvesting. However, other properties of natural pigments, such as catalytic [7] and even biochemical [8] have been proposed in the past but have been scarcely explored, in spite of their potential technological relevance.
Moreover, according to very recent works $[6,9]$ there exist evidences of nonlinear behavior of the optical properties of many natural pigments-based systems, which are just beginning to be explored. However, the thermodynamics behind such non-linear characteristics has not been addressed at all, which limits not only the understanding of the science involved, but also the potential technological applications. Accordingly, in this present paper we present a detailed analysis of the thermodynamics behind a very unique oscillatory behavior of a mixture alcohol/natural anthocyanin, which could be employed as an optical switch [9].

\section{EXPERIMENTAL BACKGROUND}

Chiu et al. [9] have prepared a mixture of ethanol with an extract of Hibiscus sabdariffa $L$, a natural anthocyanin, which is then placed in a very narrow

Corresponding author: V.M. Castaño, e-mail: vmcastano@unam.mx

(c) 2018 Advanced Study Center Co. Ltd. 


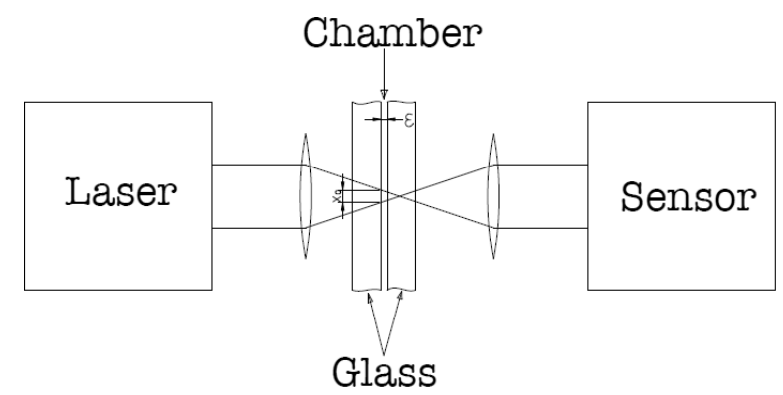

Fig. 1. Chiu's experimental setup. The laser light in the chamber has the form of a truncated cone. $\psi_{1}$, the distance between the common focus and the chamber's middle place, is varied to modify the laser intensity.

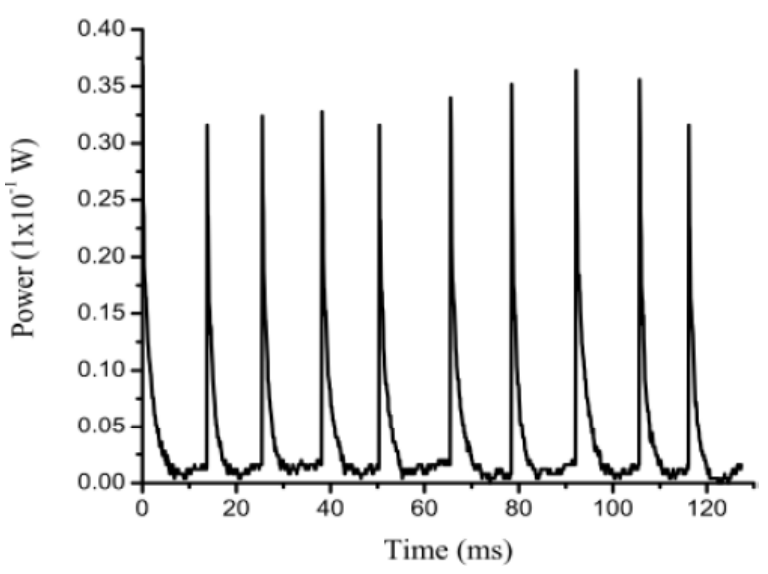

Fig. 2. Laser power recorded by sensor.

optical chamber (thickness $\varepsilon=200 \mu \mathrm{m}$ ) and submitted to a $45 \mathrm{~mW}$ laser beam. The experimental setup is shown in Fig. 1, where both lenses havea common focal length of $50 \mathrm{~mm}$. The chamber may be precisely displaced along the lenses' axis, to vary the distance from its middle plane to the lenses' common focus. The available laser light after this interaction, is gathered by the right hand lens, and its total power recorded. The laser is applied on a truncated cone, so thin that it may be approximated by a cylinder, to be called the activation cylinder, whose left radius is $r_{0}=x_{0} / 2$. Fig. 2 shows the power recorded by the sensor, i.e., of that part of the beam which is able to go through the dopped ethanol. Remark that the power presents a step-like transition from 2 to $35 \mathrm{~mW}$, that is, the system suddenly stops absorbing light. We think that this is due to a sudden vaporization of ethanol, and to remain in such state for $1 \mathrm{~ms}$, approximately, when the ethanol begins to recover its liquid state. We define our system $\Sigma$ as that part of the ethanol which was initially in the cylinder of activation, $z$ moles, for a given distance $\psi$. As it absorbs heat, its radius, which we may write as $r=r(t)$, increases, although the heat- ing zone, $r \leq r_{0}$, the activation cylinder, remains fixed. We represent the surrounding ethanol, found between $r(t)$ and $\infty$, by $\Sigma^{r}$. Since $\Sigma$ grows very fast when a part of it evaporates, it excerts a big pressure on $\Sigma^{r}$, forcing its inner boundary to move outwards, and reacting itself on $\Sigma$, due to its viscosity. Thus, $\Sigma$ must do work against $\Sigma^{r}$. Recall that $\Sigma^{r}$ is submitted to the atmospheric pressure $p_{1}=10^{5} \mathrm{~Pa}$ along its outer boundary.

In this process, the system remains liquid until, suddenly, evaporates. However, this is not the evaporation along the equilibrium line between liquid and vapor, where both pressure and temperature remain fixed, a continuous process, but of a steady rise in temperature, while the pressure remains fixed and equal to the atmospheric pressure, until a certain state is attained, when a sudden transformation from liquid to vapor is produced. We shall discuss the absorption of heat by our system in Section 4.1, and how it looses it by thermal contact with the chamber walls, in section 4.2.

\section{THERMODYNAMIC FRAMEWORK}

\subsection{Theory}

According to the classical work by Landau and Lifshitz [10], the states of the system at temperature $T$ can be represented in the volume $v$ pressure $p$ diagram, as in Fig. 3 , which is basically the original by Landau and Lifshitz, except that the curves were plotted using the van der Waals equation. The arcs of the red curve that go from $a$ to $b$ and from $e$ up to $f$, represent the isotherm at temperature $T$ for the liquid and gaseous states, respectively. Being the system in state $b$, an isothermal expansion can

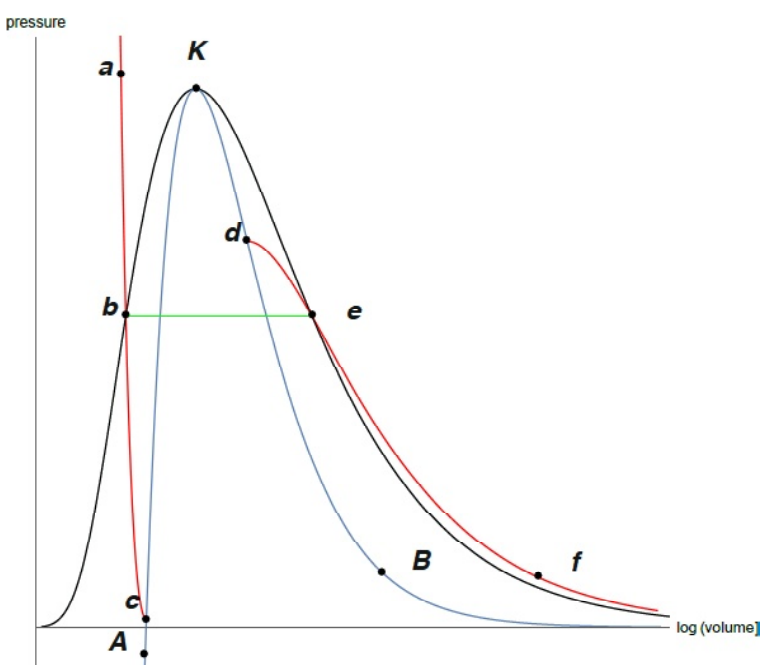

Fig. 3. Metastable states and their unstable boundary according to Landau [10]. 
produce, either a displacement along the horizontal green segment be, where the liquid is in equilibrium with its vapor, or along the arc $b c$, the superheated liquid. Similarly, at point $e$, an isothermal compression can produce either a displacement along the line ebor an overcooled vapour along arc ed. When a set of isotherms is plotted, the bell whose boundary is the black curve $b K_{e}$, where $K$ corresponds to the critical point, encloses all the states $(v ; p ; T)$, in which the system can be in one or two phases. Back to the isotherm at temperature $T$, the states found in arcs bc and de are metastable, $b$ and $e$ stable, and $c$ and $d$, impossible from the thermodynamics' standpoint. This is because the thermodynamic states must satisfy the inequality [10]:

$$
\left(\frac{\partial p}{\partial v}\right)_{T}<0
$$

This condition, however, is not fulfilled at $c$ and $d$, but rather:

$$
\left(\frac{\partial p}{\partial v}\right)_{T}=0
$$

also satisfied by all the states ${ }^{1}$ along the dark blue line $A c K d B$, which encloses all the thermodynamical states in which the system cannot exist in a single phase. Between the bell-shaped curves $A c K d B$ and $b K e$ is the region of metastable states.

\subsection{Thermodynamic modeling of the process}

According to the above, the complete process is represented in Fig. 4. The heating produced by the laser beam takes the system $\Sigma$ along the atmospheric pressure magenta line, which we can identify in the lower left corner of Fig. 4. The process begins at the far left, without label, in the initial state at temperature $T_{1}$ and atmospheric pressure $p_{1}$. The temperature rises, due to the laser heating, and the system reaches first the outer bell (black) at the state labeled $\beta$, where the zone of metastable states begins. Laser heating continues until the system reaches the inner bell (dark blue), at the state labeled $c$, with pressure $p_{c}$, molar volume $v_{c}$, and temperature $T_{c}$, of some isotherm $T_{c}$, plotted in red. Not being able to stay in one phase, the liquid spontaneously begins a decomposition of a part in its vapor ${ }^{2}$. From the thermodynamic point of view, this means

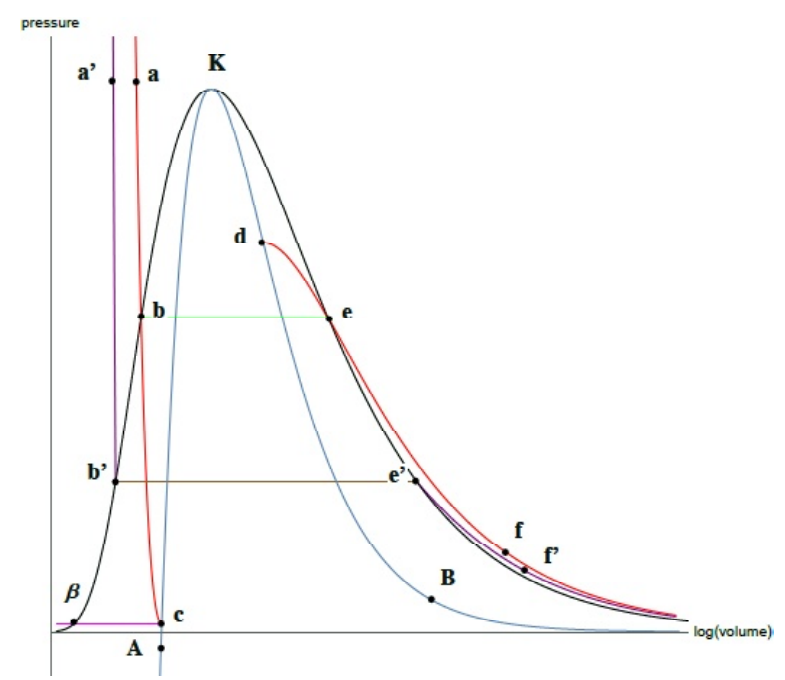

Fig. 4. The isobar be represents the equilibrium states at temperature $T_{c}$ and vapor pressure $p_{v}\left(T_{c}\right)$, while 'b'e', the corresponding states at temperature $T_{\phi}$. $\beta b K e$ and $c K d B$ are the outer and inner bells respectively.

a transition from the state $c$ to some point along the equilibrium line at temperature $T_{c}$, provided that the system is maintained at constant temperature. However, according to Chiu et al. 9], it is not the system temperature which remains constant, but the outer boundary of $\Sigma^{r}$ which is kept under atmospheric pressure $p_{1}$.

To understand the resulting process, we can decompose the volume $v_{c}$ of $\Sigma$, whose state is $c$, in volume elements. Let us consider the first one of these, which is converted to liquid and gas, both at temperature $T_{c}$ and pressure $p_{V}\left(T_{c}\right)$. Recall that the system is submitted to a lower pressure $p$, so that the produced vapor expands while pushing the inner boundary of $\Sigma^{r}$, displacing it outwards. Since the viscosity of $\Sigma^{r}$ is opposed to such expansion, the pressure against which our system produces work increases. This same analysis is repeated on all the remaining elements into which $v_{c}$ was decomposed, giving rise to a rapid process, without heat exchange, and therefore, adiabatic, which allows us to deduce that the temperature falls from $T$, to a certain average value $T_{\phi}$, while the process will be characterized also by a certain average value $p_{\phi}$, of the pressure against which $\Sigma$ realizes work, with $p_{1}<p_{v}\left(T_{\phi}\right)<p_{v}\left(T_{c}\right)$, where $p_{v}(T)$ denotes the vapor pressure of the thermodynamic system at temperature $T$. The $T_{\phi}$ isotherm in Fig. 4 joins the states

${ }^{1}$ To be called unstable in the sequel.

${ }^{2}$ This decomposition may also happen in the metastable states, between $b$ and $c$, provided that a gas, different from its vapor, is diluted in the liquid. 
$a^{\prime} b^{\prime} e^{\prime} f$ ', while the state $\phi$, not shown, must be in the horizontal segment $b$ ' $e$ '.

According to the above, the system spontaneously jumps not to the liquid vapor equilibrium line at temperature $T_{c}$, but to that of a cooler (purple) isotherm at temperature $T_{\phi}$, due to the adiabatic expansion. Since the system falls to a liquid-vapor equilibrium state at that temperature, the reached state is at some point in the horizontal segment $b^{\prime} e^{\prime}$, the isobar of coexistenceliquid-vapor, at pressure $p_{\phi}=p_{v}\left(T_{\phi}\right)$. The volume $v_{\phi}$ is made up of a certain amount $\xi$ of vapor, whose molar volume is $v_{g}\left(T_{\phi}\right)$ and $1-\xi$ of liquid, whosemolar volume is $v_{\lambda}\left(T_{\phi}\right)$. Our objective is to calculate the amount $T_{\phi}$ of vapor produced as a function of the temperature $T_{\phi}$, which is unknown.

From the state $\left(v_{\phi} ; p_{\phi}\right)$, while $\Sigma^{r}$ is submitted to atmospheric pressure on its outer boundary, $\Sigma$ must fall to a state in the atmospheric isobar, where the thermal contact of ethanol with the walls of the container, cools it down, reducing its volume along the atmospheric isobar, the vapor condenses down to the $\beta$ state, where a new cycle begins.

In what follows, a subscript $\phi$ will be assigned to the thermodynamic variables in that state. According to the first law of thermodynamics:

$$
\Delta u=u_{\phi}-u_{c}=q+\omega,
$$

where $q=0$ is the absorbed heat and $w$, the work done on the system. Assuming, during this expansion, that the system works against a pressure $p_{\phi}$, we can write:

$\omega=-p_{\phi}\left(v_{\phi}-v_{c}\right)$.

In this way, $\Sigma$ pushes its surroundings, but only does work on $\Sigma^{r}$, since we assume that the glass walls of the chamber remain fixed. $\phi$, the resulting state, a liquid and vapor mixture at temperature $T_{\phi}$ has a molar volume $v_{\phi}$ and molar energy $u_{\phi}$ given by

$$
\begin{aligned}
& v_{\phi}=\xi\left(T_{\phi}\right) v_{g}\left(T_{\phi}\right)+\left(1-\xi\left(T_{\phi}\right)\right) v_{l}\left(T_{\phi}\right), \\
& v_{\phi}=\xi\left(T_{\phi}\right) u_{g}\left(T_{\phi}\right)+\left(1-\xi\left(T_{\phi}\right)\right) u_{l}\left(T_{\phi}\right),
\end{aligned}
$$

The system volume increase produced by evaporation, suddenly decreases the absorption of laser light, as we will show in Section 4.1, resulting in a discontinuous jump in the quantity of light that crosses the activation cylinder. Then the system starts to be cooled down by its contact with the walls, until it is condensed again, thus beginning a new cycle.

\subsection{Evaluation of ethanol properties from van der Waals equation}

To analyze this process, we will describe the ethanol using the van der Waals equation, which, although only depends on two parameters, it does contain the germ of both stable and unstable states, providing thus a good picture of the real process.

$p(a, b, T, v)=\frac{R T}{v-b}-\frac{a}{v^{2}}$.

The gas constant $R$ is given by

$R=8.31446 \mathrm{~kg} \mathrm{~m}^{2} \mathrm{~s}^{-2} \mathrm{~K}^{-1} \mathrm{~mol}^{-1}$,

while the $a$ and $b$ of Eq. (7) are given by [11]

$$
\begin{aligned}
& a=1.218 \mathrm{~kg} \mathrm{~m}^{6} \mathrm{~Pa} \mathrm{~mol}^{-2}, \\
& b=8.407 \times 10^{-5} \mathrm{~m}^{3} \mathrm{~mol}^{-1} .
\end{aligned}
$$

The unstable states satisfy Eq. (2), which can be written for a van der Waals gas as:

$\frac{\mathrm{d} p}{\mathrm{~d} v}=\frac{2 a}{v^{3}}-\frac{R T}{(v-b)^{2}}=0$.

Deducing $R T$ and substituting it in Eq. (7), we obtain:

$p_{e}(a, b, v)=\frac{a(v-2 b)}{v^{3}}$.

At the state $c$, mentioned in Section 3, the pressure is $p_{1}$, i.e, the atmospheric. The equation $p_{e}(a, b, v)=p_{1}$ has three real roots. The negative one will be neglected. The smallest and biggest of the other two roots correspond to points $c$ and $d$ of Fig. 3. Form here, it follows that the volume in $c$ is $v_{c}=$ $1.685 \times 10^{-4} \mathrm{~m}^{3} \mathrm{~mol}^{-1}$, whereas the temperature $T_{c}=$ $436.6 \mathrm{~K}$ can be deduced from Eq. (11).

At that point, the atmospheric isobar is tangent to the minimum of the van der Waals isotherm, at temperature $T_{c}$, whose molar volume is $v_{c}$. The vapor pressure associated with this isotherm, which we have calculated using Maxwell's rule of equal areas, turns out to be $p_{v}\left(T_{c}\right)=3.148 \times 10^{6} \mathrm{~Pa}$, while the molar volumes of the liquid $v_{l}$ and vapor $v_{g}$ are $1.38 \times 10^{-4}$ and $8.08 \times 10^{-4} \mathrm{~m}^{3} \mathrm{~mol}^{-1}$, respectively.

There is, however, a problem. According to Eq. (7), and the parameters corresponding to ethanol, Eqs. (9) and (10), if we calculate vapor pressure of ethanol at $T=298.4 \mathrm{~K}$, we obtain, using Maxwell's rule, $p_{v}(298.4)=4.45 \times 10^{5} \mathrm{~Pa}$. However, the experimental value is, according to Schroeder et al. [12], 
$p_{v}(298.4)=8 \mathrm{kPa}$, almost 56 times smaller. The scheme we have adopted is not useful at room temperature, very far from the critical temperature ${ }^{3}$.

\subsection{Evaluation of ethanol properties from a modified van der Waals equation}

Instead of working with the van der Waals equation for ethanol, with the values of constants $a$ and $b$ given by Eqs. (9) - (10) used in the previous section, we will propose new ones, since those calculated from the properties at the critical state fail to predict thermodynamic properties at room temperature.

Since the experiment starts at room temperature, we shall investigate what the coefficients should be at that temperature. In accordance with Schroeder et al. [12], at $T=298.4 \mathrm{~K}$, as indicated above, the pressure of ethanol is $p_{v}(298: 4)=8 \mathrm{kPa}$, while its density in the liquid and vapor states is 784.8 and $0.1494 \mathrm{~kg} \mathrm{~m}^{-3}$, respectively. Since the molar mass of ethanol is $46.07 \mathrm{~g} \mathrm{~mol}^{-1}$, we can deduce that the molar volumes of ethanol are $5.876 \mathrm{x}$ $10^{-5}$ and $3.084 \times 10^{-1} \mathrm{~m}^{3} \mathrm{~mol}^{-1}$. From these data, we can calculate that the parameter $b$ of van der Waals should be

$$
b=5.275 \times 10^{-5} \mathrm{~m}^{3} \mathrm{~mol}^{-1} .
$$

In order to determine the parameter a we can impose, either that the preceding values are produced at temperature $T=298.4$ or that the vapor pressure is $p_{v}=8 \mathrm{kPa}$. We preferred to impose the temperature, which has led us to to adopt the value

$$
a=1.436 \mathrm{~m}^{6} \mathrm{~Pa} \mathrm{~mol}^{-2} \text {. }
$$

From the given values for $a$ and $b$, we can deduce that the vapor pressure must take the value $p_{v}$ $=8031.68 \mathrm{~Pa}$, which almost coincides with the experimental value. The chosen values for $a$ and $b$ produces good results around $T=298.4$. However, as soon as we tried to calculate the temperature of the isotherm whose minimum, the point $c$, is tangent to the atmospheric isobar, we obtained a temperature above the critical temperature, which cannot be. We took then the decision to adopt temperature-dependent coefficients, leading to a Modified van der Waals Equation of State (MVDW), that is, $a=a(T)$ and $b=b(T)$, given by:

$$
a(T)=a\left(T_{1}\right)+\left(T-T_{1}\right) \times \frac{a\left(T_{2}\right)-a\left(T_{1}\right)}{T_{2}-T_{1}},
$$

$b(T)=b\left(T_{1}\right)+\left(T-T_{1}\right) \times \frac{b\left(T_{2}\right)-b\left(T_{1}\right)}{T_{2}-T_{1}}$,

where $T_{1}=298.4 \mathrm{~K}$, is the ambient temperature and $T_{2}=516.296 \mathrm{~K}$, the critical temperature. This allowed to reproduce correctly not only the extremes, but also the intermediate temperatures. It is convenient to define the temperature rate of change of such constants:

$D a=\frac{a\left(T_{2}\right)-a\left(T_{1}\right)}{T_{2}-T_{1}}, \quad D b=\frac{b\left(T_{2}\right)-b\left(T_{1}\right)}{T_{2}-T_{1}}$.

from this, the MVDW equation of state is:

$p(T, v)=\frac{R T}{v-b(T)}-\frac{a(T)}{v^{2}}$,

where $a(T)$ and $b(T)$ are given by Eqs. (15)-(16). In the unstable states, such as $c$, Eq. (2) reduces in the MVDW to:

$\frac{\mathrm{d} p}{\mathrm{~d} v}=\frac{2 a(T)}{v^{3}}-\frac{R T}{(v-b(T))^{2}}=0$,

from which $T$ can be determined:

$T=\frac{a(T)}{v^{3}} \frac{2(v-b(T))^{2}}{R}$,

and substituted in Eq. (18):

$p_{e}=\frac{a(T)(v-2 b(T))}{v^{3}}=p_{1}$.

As the pressure in state $c$ must match the atmospheric, this equation establishes then a connection between $v$ and $T$ that we can write as $v=v(T)$, to be substituted Eq. (20), producing thus an equation for $T$. If, as we have said, we set $p_{e}=p_{1}$ in Eq. (21), the thermodynamical coordinates of state $c$ are:

$$
\begin{aligned}
& T_{c}=479.7 \mathrm{~K}, v_{c}=1.579 \times 10^{-4} \mathrm{~m}^{3} \mathrm{~mol}^{-1}, \\
& p_{v}\left(T_{c}\right)=3.686 \times 10^{6} \mathrm{~Pa},
\end{aligned}
$$

where we have included $p_{v}\left(T_{c}\right)$, calculated using Maxwell's law of equal areas. For state $\beta$ we have:

$$
\begin{aligned}
& T_{\beta}=344.9 \mathrm{~K}, v_{\beta}=6.935 \times 10^{-5} \mathrm{~m}^{3} \mathrm{~mol}^{-1}, \\
& p_{v}\left(T_{\beta}\right)=1.0 \times 10^{5} \mathrm{~Pa} .
\end{aligned}
$$

Fig. 5 includes the new information. Notice that the liquid-vapor equilibrium at temperature $T=298: 4 \mathrm{~K}$, the isobar at $p=p_{1}$, is almost confused with the

${ }^{3}$ The van der Waals parameters are calculated normally from the data at the critical state. 


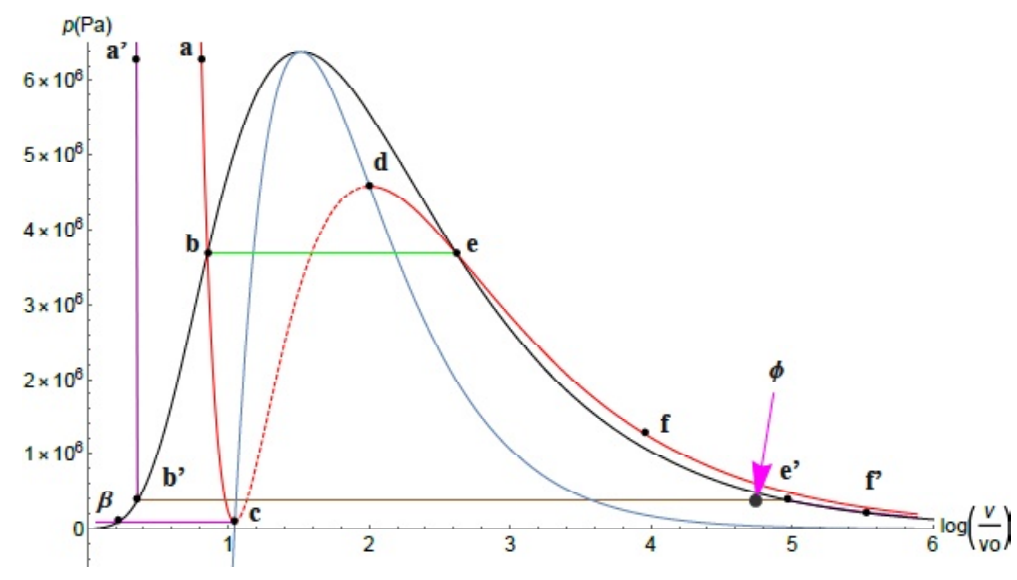

Fig. 5. The laser heats the system along the atmospheric isobar, going first through state $\beta$, then to state $c$ located in the $479.9 \mathrm{~K}$ isotherm. Sudden evaporation occurs there taking the system to state $\phi$. Subsequent cooling due to heat exchange with the chamber walls liquefies the system bringing it back to state $\beta$ where a new cycle begins.

axis of the abscissas, a merit of the approach proposed in this Section.

As we explained in Section 3, the system jumps from state $c$ not to a point of the liquid-vapor equilibrium line at temperature $T_{c}$, but to a state found in the $b^{\prime} e^{\prime}$ isobar, the equilibrium line of the $T_{\phi}$ isotherm. We still have no idea whatsoever of the temperature $T_{\phi}$, but we do want to calculate at which point, as a function of $T_{\phi}$, the system ends. For that we need the molar energy of ethanol depending on the molar volume $v$ and the absolute temperature $T$.

\subsection{Molar energy $u=u(T, v)$}

We take as a starting point the following thermodynamic identity, the basis of which is found, for example, in section 16 of the Landau textbook [10].

$$
\mathrm{d} u=\left(\frac{\partial u}{\partial T}\right)_{v} \mathrm{~d} T+\left[T\left(\frac{\partial p}{\partial T}\right)_{v}-p\right] \mathrm{d} v .
$$

Let us calculate $(\partial p / \partial T)_{v}$ from Eq. (18), with the coefficients $a(T)$ and $b(T)$ given by Eqs. (15), (16), and (17).

$$
\begin{aligned}
& \mathrm{d} u=\left(\frac{\partial u}{\partial T}\right)_{v} \mathrm{~d} T+\left\{\frac{a\left(T_{1}\right)-T_{1} D a}{v^{2}}+\right. \\
& \left.\frac{R D b T^{2}}{\left[v-\left[\left(b\left(T_{1}\right)+\left(T-T_{1}\right) D b\right)\right]\right]^{2}}\right\} d v .
\end{aligned}
$$

We thus propose for the molar energy $u(T, v)$ :

$$
\begin{aligned}
& u(T, v)=f(T)-\frac{a\left(T_{1}\right)-T_{1} D a}{v^{2}}- \\
& \frac{R D b T^{2}}{v-\left[b\left(T_{1}\right)+\left(T-T_{1}\right) D b\right]},
\end{aligned}
$$

where $f(T)$ is a function of the temperature $T$ to be determined from experimental results on the constant volume specific heat $c_{v}=(\partial u(T, v)=\partial T)_{v}$ :

$$
c_{v}(T, v)=-\frac{D b^{2} R T^{2}}{[v-b(T)]^{2}}-\frac{2 D b R T}{v-b(T)}+f^{\prime}[T]
$$

Since this function has a second order pole in $v=$ $b(T)$, and the liquid molar volume $v_{1}(T)$ is close to $b(T)$, we will calculate the preceding function in the vapor molar volume $v_{g}(T)$. $f^{\prime}(T)$ is thus given by:

$$
\begin{aligned}
& f^{\prime}(T)=c_{v}\left(T, v_{g}(T)\right)+\frac{D b^{2} R T^{2}}{\left[v_{g}(T)-b(T)\right]^{2}}- \\
& \frac{2 D b R T}{\left[v_{g}(T)-b(T)\right]} .
\end{aligned}
$$

Using the data for $c_{v}\left(T, v_{g}(T)\right)$ reported by Schroeder [12], we can plot $f(T)$ in Fig. 6 . Since the temperature of our system varies between 298.4 and $480 \mathrm{~K}$, we can approximate $f(T)$ by the straight line:

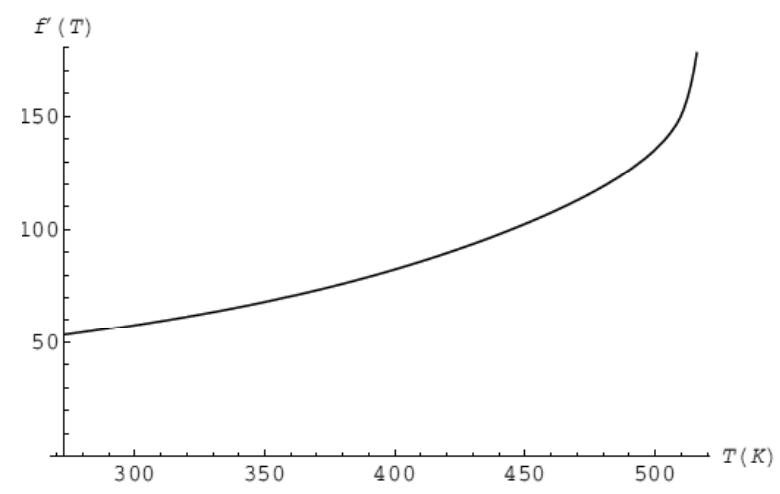

Fig. 6. $f(T)$, Eq. (28), calculated from the constant volume specific heat from Schroeder et al. [12]. 


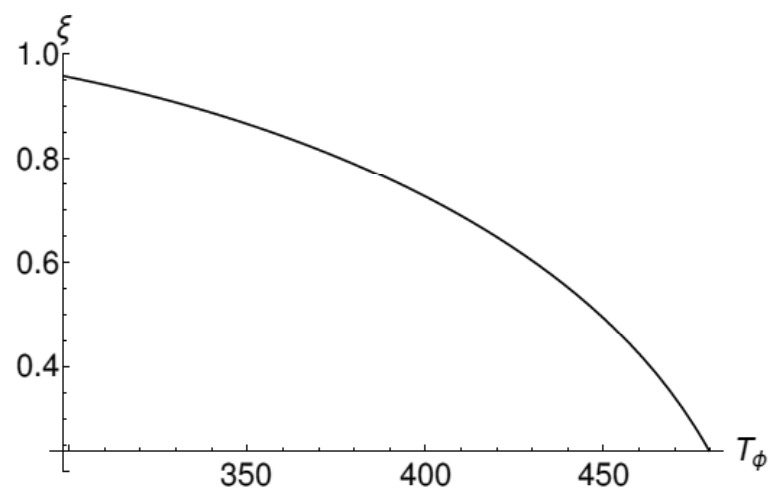

Fig. 7. Molar fraction $\xi$ transformed in vapor in the transition $c \rightarrow \phi$ as a function of temperature $T_{\phi}$.

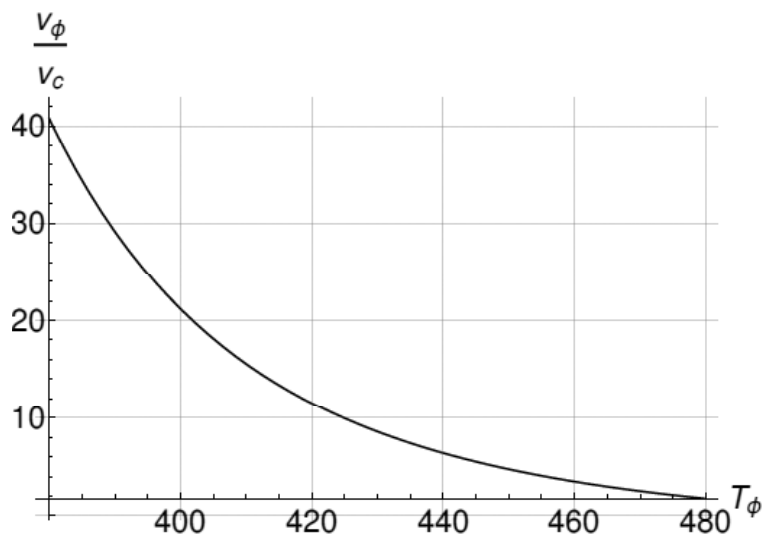

Fig. 8. Ratio of volume $v_{\phi}$ to volume $v_{c}$ as function of temperature $T_{\phi}$.

$$
f^{\prime}(T)=-45.11+0.3265 T \text {. }
$$

\subsection{Transition from state $\mathbf{c}$ to state $\phi$}

We write the first law, Eq. (3), taking into account that $q=0$, since we have assumed the process to be so fast that the system is not able to exchange heat with its surroundings, and that work is given by Eq. (4):

$$
u_{\phi}-u_{c}=-p_{\phi}\left(v_{\phi}-v_{c}\right) \text {, }
$$

where $v_{\phi}$ is given by Eq. (5) and $u_{\phi}$ by Eq. (6). From the resulting identity we can deduce $\xi$, the fraction of vapor, as a function of $T_{\phi}$ :

$$
\xi\left(T_{\phi}\right)=\frac{u_{c}+p_{\phi} v_{c}-h_{l}\left(T_{\phi}\right)}{h_{g}\left(T_{\phi}\right)-h_{l}\left(T_{\phi}\right)},
$$

where we have defined the enthalpy as $h=u+p v$, to be calculated either in $v=v_{g}(T)$, the molar vapor volume, or in $v=v_{l}\left(T_{\phi}\right)$, the molar liquid volume, obtaining thus $h_{g}$ and $h_{l} . \xi\left(T_{\phi}\right)$ is plotted in Fig. 7, where we can see that the lowest vapor production occurs when there is no decrease in temperature, i.e. when $T_{\phi}=T_{c}$. Until now we know nothing of $T_{\phi}$, except that it must be below $T_{c}$, in view of the continuous adiabatic expansion as the superheated liquid evaporates. This result allows us to completely locate the state $\phi$ for a given value of the temperature $T_{\phi}$. We can see that $T_{\phi}$ must be above room temperature $T_{1}$. The volume $v_{\phi}$ can be calculated as a function of $\xi$ :

$$
v_{\phi}=\xi\left(T_{\phi}\right) v_{g}\left(T_{\phi}\right)+\left[1-\xi\left(T_{\phi}\right)\right] v_{l}\left(T_{\phi}\right),
$$

Fig. 8 shows the ratio of $v_{\phi}=v_{c}$, which measures the volume increase due to evaporation, only from $T_{\phi}=$ $380 \mathrm{~K}$ to $T=T_{c}$ due to the discussion presented in Section 3.1. At $T=T_{c}$, when there is no temperature drop, this coefficient is minimum and equal to 1.77 .

\subsection{If the system evolution went through equilibrium states}

If we apply a very low power laser radiation, the system evolves along states of thermodynamic equilibrium, always under atmospheric pressure. It starts at the left end $\left(v_{1}, p_{1}\right)$, of the magenta line, where $v_{1}$ $=5.870 \times 10^{-5} \mathrm{~m}^{3} / \mathrm{mol}$, and room temperature $T_{1}$, warming up to state $\beta$ at $\left(v_{\lambda}\left(T_{\beta}\right), p_{1}\right)$, cf. Fig. 5. As the laser beam continues to heat the system, the evaporation starts at this state, moving to the right, always under atmospheric pressure, until the magenta line cuts the external bell $\beta$ b'bee', when all the liquid is evaporated.

\subsection{Return to initial conditions}

The return to the initial state occurs along a path that we can draw in the volume pressure plane. The system is now in a state at temperature $T_{\phi}$ and pressure $p_{v}\left(T_{\phi}\right)$. The process of evaporation is over, $\Sigma^{r}$ ceases to react via the viscosity, the vapor pressure drops rapidly to the atmospheric level. The system is then cooled by contact with the glass walls of the chamber, the vapor becomes liquid and the system attains state $\beta$. If now the laser is on, as we assume, the system will not continue to the initial state, but begin a new cycle, heading now towards state $\mathrm{c}$.

\section{HEAT ADDED TO THE SYSTEM}

\subsection{Power absorbed from the laser beam}

Let $\sigma$ be the cross section that allows us to calculate the probability that a unit of the dissolved natural pigment captures a photon of frequency $\omega$ and 
absorbs its energy. Since the laser frequency is fixed, $\sigma$ is a constant ${ }^{4}$. Denoting by $q_{j}$ the quantity of pigment in a mole of ethanol, there are $q_{j} N_{\mathrm{a}}$ dispersing units in a mole, where $N_{\mathrm{a}}$ is Avogrado's number.

Let $v_{1}$ be the molar volume of ethanol at pressure $p_{1}$ and temperature $T_{1}$, that is, the standard pressure and temperature conditions. The volume of the activation cylinder is, cf. Section $2, \pi r_{0}^{2} \varepsilon$, so that, $\Sigma$, the thermodynamic system consists of $z$ moles,

$z=\frac{\pi r_{0}^{2} \varepsilon}{v_{1}}$.

The number of dispersing centers is $z q_{j} N_{\mathrm{a}}$. However, only the fraction

$$
z q_{j} N_{a} \frac{\pi r_{o}^{2}}{\pi r^{2}(t)},
$$

are within the cylinder of activation, where $r(t)$ is the radius of the system $\Sigma$ at time $t$. The accumulated area of these centers is

$$
\frac{\sigma z q_{j} N_{a} r_{o}^{2}}{r^{2}(t)}
$$

so, the probability of a photon being absorbed is

$$
\frac{\sigma z q_{j} N_{a}}{\pi r^{2}(t)}
$$

provided this number is smaller or equal to 1 , being 1 otherwise. If we now send $n$ photons per second inside the area $\pi r^{2}$, the number of absorbed photons is given by:

$$
\frac{\sigma z q_{j} N_{a} \dot{n}}{\pi r^{2}(t)}
$$

so, the absorbed power is:

$$
\dot{Q}_{l}=\frac{(\dot{n} \hbar \omega)\left(\sigma z q_{j} N_{a}\right)}{\pi r^{2}(t)} .
$$

The preceding result assumes that there are no loses between emission at the laser and its arrival to $\Sigma$. Let $\eta_{b}$ and $\eta_{a}$ stand for the fractions of power lost before and after $\Sigma$. The power recorded at the sensor is given by:

$$
P_{s}=(\dot{n} \hbar \omega)\left(1-\eta_{b}\right)\left(1-\frac{\in \sigma z q_{j} N_{a}}{V_{\Sigma(t)}}\right)\left(1-\eta_{a}\right),
$$

where we have defined the volume of $\Sigma$ as $V_{\Sigma}=\pi r^{2}(t) \varepsilon$. The cross section $\sigma$ introduced in this section solves two different problems. The fist one, connected with the probability to scatter and absorb a photon from the laser beam, is used in all the equations in this section, except Eq. (39), where it is connected with the probability to scatter. In the sequel, we assume that the difference between these cross sections is negligible.

\subsection{Power loss}

The partially evaporated ethanol at temperature $T$ is in contact with the walls whose temperature is not far from $T_{1}$, the room temperature. We assume that the heat exchange can be described by Newton's cooling law, i.e., that he heat flux $Q_{\mathrm{e}}$ directed to the walls of the NLS chamber can be approximated by

$\dot{Q}_{e}=-2 \gamma \pi r^{2}(t)\left(T-T_{1}\right)$,

where $\gamma$ is a constant.

This exchange, very important when the system is evaporated, having thus a large volume, and exchanging a large amount of heat from the surroundings, drives the system $\Sigma$ from state $\phi$, to decrease its pressure to the atmospheric level, and finally to condense it, to return to state $\beta$.

\section{THEORY AGAINST EXPERIMENTAL RESULTS}

\subsection{Measured power}

Fig. 2 shows nine cycles extending along a time interval of $117 \mathrm{~ms}$, with an average period of $13 \mathrm{~ms}$. Remark the digital uncertainty in the lower part of the figure, a fact supporting a very strong interaction between the laser beam and the doped ethanol. These cycles show a certain reproducibility whose variations could be connected with temperature fluctuations between different cycles. We can locate precisely the time when the recorded power changes discontinuously, from a low value of less than 5 to more than $30 \mathrm{~mW}$.

\subsection{Recovered power analysis}

The key to analyse Fig. 2, the recorded laser beam power as a function of time, is Eq. (39) which shows that large values of the recorded power $P_{s}$ are connected with large values of the system volume $V_{\Sigma}$. Thus, the minimum value of each one of the nine

${ }^{4}$ The states of such particles are temperature dependent. In a sharper analysis, this fact should be taken into account. 
Table 1. Numerical data read from Fig. 2. $P_{M}$ stands for the largest recorded power, while $P_{c}$ and $P_{\phi}$ are the corresponding values of the power recorded at states $\mathrm{c}$ and $\phi$, respectively.

\begin{tabular}{ll}
\hline label & $(\mathrm{ms}, \mathrm{mW})$ \\
\hline$P_{M}$ & $(85.15,36.43)$ \\
$P_{c}$ & $(96.42,1.440)$ \\
$P_{\phi}$ & $(93.40,0.7582)$ \\
\hline
\end{tabular}

cycles should correspond with the system volume at state $\beta$, while the power immediately before the upward discontinuity, with the system volume at state $c$. Following the same idea, we identify the largest power recorded with the largest system volume, when an important amount of liquid has been transformed into vapor. Focusing our attention on the largest signal $P_{M}$ and the following cycle, the following data coordinates (time (ms), power(W)) have been read off from Fig. 2. Assuming that $V_{\Sigma}$ is so large for state producing the largest signal PM that Eq. (39) may be approximated by:

$$
P_{s}=(\dot{n} \hbar \omega)\left(1-\eta_{b}\right)\left(1-\eta_{a}\right) \text {, }
$$

where $n h \omega=0.045 \mathrm{~W}$, we can deduce that

$$
\frac{P_{M}}{\dot{n} \hbar \omega}=\frac{0.03643}{0.045}=0.81 .
$$

We can thus write:

$$
\frac{\varepsilon \sigma z q_{j} N_{a}}{V_{\Sigma}(t)}=1-\frac{P_{s}(t)}{P_{M}} .
$$

Calculating this ratio for $P_{s}=P_{c}$ and $P_{s}=P_{\phi}$, we obtain

$$
\frac{V_{\Sigma}(\phi)}{V_{\Sigma}(c)}=\frac{0.9605}{0.02388}=40.22
$$

According to the theory here developed, this ratio should match $v_{\phi}=v_{c}$, plotted in Fig. 8 as a function of $T_{\phi}$, allowing us to identify $T_{\phi}=380.4 \mathrm{~K}$, the system having performed a work against a mean pressure of $p_{v}\left(T_{\phi}\right)=3.760 \times 10^{5} \mathrm{~Pa}$. The fraction of liquid having vaporized is $\xi(380.4)=0.7886$. At the given temperature $T_{\phi}$, the molar volumes of the liquid and vapor phases are $7.912 \times 10^{-5}$ and $8.033 \times$ $10^{-3} \mathrm{~m}^{3} / \mathrm{mol}$ respectively. The volume $v_{\phi}$ of state $\phi$ according to Eq. (32) is $v_{\phi}=6.352 \times 10^{-3} \mathrm{~m}^{3} / \mathrm{mol}$.

Let us apply Eq. (43) to state $V_{\Sigma, \mathrm{c}}=z v_{c}$, using the molar fraction $z$ given by Eq. (33), initially found in the activation cylinder:

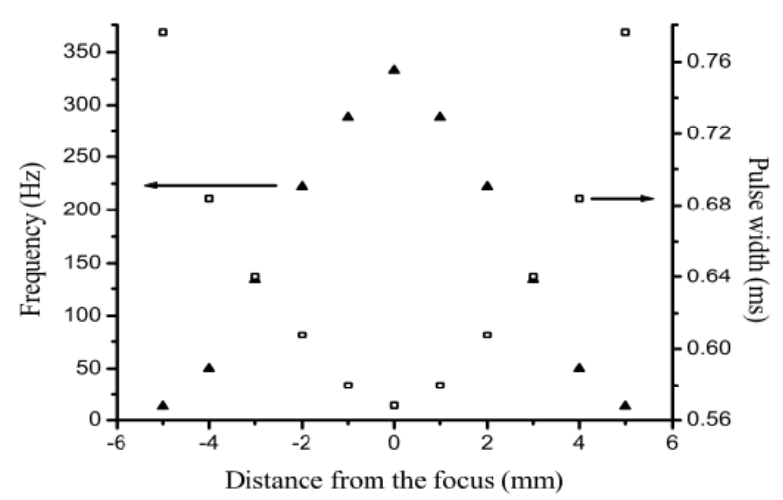

Fig. 9. Pulse repetition rate (solid triangle) and pulse width (empty square) as a function of $\psi$, the distance between the chamber middle plane and the lenses' common focus. Figure taken from the work of Chiu et al. [9].

$$
\frac{\sigma q_{j} N_{a} \varepsilon}{v_{c}}=1-\frac{P_{c}}{P_{M}},
$$

which we may solve to determine $\sigma q_{j}=1.259 \times 10^{-24}$.

\subsection{Pulse repetition rate and pulse width}

As we have seen, the transition to state $\phi$ occurs as the system state reaches state $\mathrm{c}$, the temperature decreasing thus to $T_{\phi}=395.34 \mathrm{~K}$, while the volume increases up to $v_{\phi}=6.352 \times 10^{-3} \mathrm{~m}^{3} / \mathrm{mol}$ due to adiabatic expansion and work is performed against $\Sigma$. Thence, to cool down to room temperature and condense down to state $\beta$, where the cycle restarts. The time required to cool from $T_{\phi}$ to $T_{\beta}$ should be approximately constant. In fact, we can measure it directly, although approximately, by the time width of the pulses in Fig. 2, since these pulses are visible only while the system is cooling by thermal contact with the walls.

This time width, according to Fig. 9, varies from 0.56 to $0.77 \mathrm{~ms}$, depending only in the distance $\psi$ between the chamber's middle plane and the lenses' common focus. The very small variation observed is due to the activation cylinder volume, which grows as the absolute value of $\psi$. When this volume is large, the heating time increases, a fact which also increases the temperature of all the system's boundaries, i.e., the temperature of the inner $\Sigma^{r}$ boundary and the temperature of the glass wall, lowering thus the boundaries' efficiency to cool the system. According to this plot, the pulse repetition rate decreases from $350 \mathrm{~Hz}$, when $\psi=0$, down to only $10 \mathrm{~Hz}$, when $\psi=5 \mathrm{~mm}$, the corresponding period (inverse of pulse repetition rate) varies from 


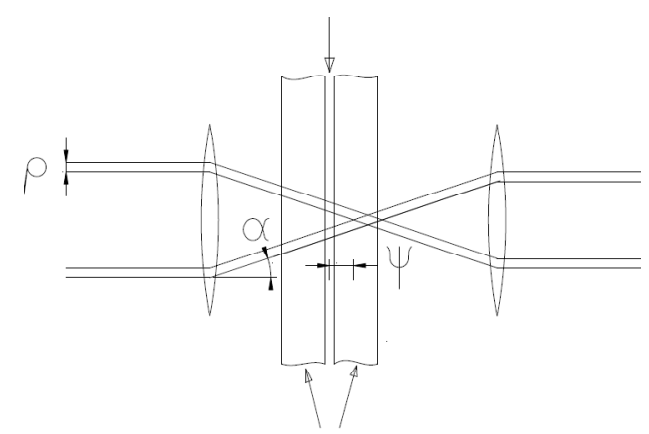

Fig. 10. Distance $\psi$ from the chamber middle plane and the common focus (of left and right hand side lenes $l_{l}$ and $l_{r}$ ). The laser beam is focused onto the disc, centered in the common focus of radios $\rho$.

2.86 to $83 \mathrm{~ms}$. This means that the heating time increases almost 40 times. We think that the cooling time must be approximately $1 / 375 \mathrm{~s}$, since 375 is the largest observed pulse repetition rate. Let us call $\tau_{1}$ the cooling time. Our object now is to calculate the warm up time $\tau_{2}$ to take the system from $T_{\beta}$ to $T_{\mathrm{c}}$ from the process' thermodynamics.

\subsection{Estimation of the warm up time $\tau_{2}$}

Since the system $\Sigma$ must change from state $\beta$ to state $c$, the molar energy must change from $u\left(T_{\beta}\right.$, $\left.v_{\beta}\right)$ to $u\left(T_{c}, v_{c}\right)$. The energy difference may be calculated using Eq. (26), which should then be multiplied by $z$, the number of moles initially enclosed in the activation cylinder, given by Eq. (33). We should, however, work a more reliable expression for the activation volume. The main difficulty arises because, in the classical approach, the left hand side lens focuses the laser beam into a point and this assumption could be false. Somehow, this lens focuses the laser beam not on a point but on a circular disc of radius $\rho$. We propose the model shown in Fig. 10, where we have depicted the distance $\psi$, the angle ${ }^{5} \alpha$, and the previously mentioned radius $\rho$. We have drawn four rays, the two inner ones in the left hand side, the classical rays, are directed to the focus of the left lens, that is, on one point. We have then drawn a circle of radius $\rho$ centered in that point, not shown, which is then used to draw the two outer rays. We have thus two cones, the first connected with the classical idea of a point focus, while the second, to account for the real zone. The activation cylinder is the intersection of the external cone with the chamber. When $\psi=0$, we have two truncated cones, and it is the intersection of this figure with the chamber which constitutes the activation cylinder. According to Fig. 10, our system is a slice of a cone ${ }^{6}$ and its volume depends on $\psi$. Using $\varepsilon / 2$ as the unit of length, we can write $\psi=$ $v(\varepsilon / 2)$ and $\rho \cot \alpha=\kappa(\varepsilon / 2)$. In terms of $\kappa$ and $v$, the volume $v_{\Sigma}(v, \kappa)$ is given by:

$v_{\Sigma}(v, \kappa)=\frac{\varepsilon^{3} \tan ^{2} \alpha}{12} \Theta(v, \kappa)$,

where $\Theta(v, \kappa)$ is defined by

$\Theta(v, \kappa)=\left\{\begin{array}{ll}1+3 \kappa+3 \kappa^{2} & \text { if } v=0 \\ 1+3(\kappa+v)^{2} & \text { if }|v| \geq 1\end{array}\right.$.

We can thus write, approximately, that

$\dot{Q}_{l} \tau_{2}=\left[h\left(T_{c}, v_{c}\right)-h\left(T_{\beta}, v_{\beta}\right)\right] z$,

where $h(T, v)$ is the enthalpy defined immediately after Eq. (31). Writing $Q$, with the help of Eq. (39) and solving for $\tau_{2}$ we obtain:

$\tau_{2}=\frac{\left[u\left(T_{c}, v_{c}\right)-u\left(T_{\beta}, v_{\beta}\right)\right] \varepsilon^{2}}{12 N_{a} \dot{n} \hbar \omega \eta_{a}} \frac{\tan ^{2} \alpha}{\sigma q_{j}} \Theta(v, \kappa)$.

We have written $\tau_{2}$ as the product of three factors. Since we only know the numerical value of the first, we shall write $\tau_{2}=\mu \Theta(v, \kappa)$. The period of a full cycle is given by $\tau_{1}+\tau_{2}$, while the pulse repetition rate $F$ is given by

$$
F=\frac{1}{\tau_{1}+\mu \Theta(v, \kappa)} .
$$

We have adjusted, by means of a least squares fit, the numerical value of the three variables $\tau_{1}, \mu$ and $\kappa$, to the set of numerical values that can be read off from Fig. 9. These values are:

$\tau_{1}=2.829 \mathrm{~ms} ; \kappa=3.518 \times 10^{-9} ;$

$\mu=2.155 \times 10^{-6}$.

Since $\rho \cot \alpha=\kappa(\varepsilon / 2)$, we obtain for $\rho=\kappa \tan \alpha$ $(\varepsilon / 2)=2=5 \times 10^{-15} \mathrm{~m}$, almost as small as the size of a proton! If we set $\kappa=0$, we obtain the same fit. Our fit shows that the classical approximation $\rho=0$ is good. Inserting

$\tau_{1}=2.829 \mathrm{~ms} ; \kappa=0 ; \mu=2.155 \times 10^{-6}$.

${ }^{5} \tan \alpha=($ radius of the incident laser beam $) /$ focal distance.

${ }^{6}$ Two cones when $\psi=0$. 


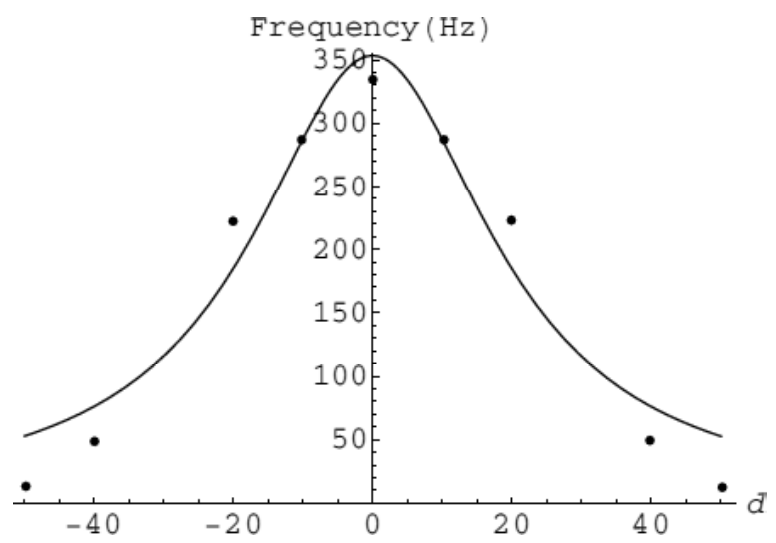

Fig. 11. Pulse repetition rate as a function of $v$, given by Eq.(50), with the numerical values of $\tau_{1}, \kappa$ and $\mu$ given by Eqs. (51).

in Eq. (50), the pulse repetition rate $F$ may be plotted as a function of $n$, shown in Fig. 11, where the experimental results are included?.

\subsection{Estimation of the molar fraction $\boldsymbol{z}=\pi \boldsymbol{r}_{0}^{2} \varepsilon / \boldsymbol{v}_{\mathbf{1}}$}

Chiu et al. [9] do not give explicit information about $r_{0}$. For this reason, we shall figure out, approximately its numerical value from thermodynamic considerations. The molar heat absorbed to go from state $\beta$ to state $c$ along the atmospheric isobar is

$$
\begin{aligned}
& \Delta Q=\left(u\left(T_{c}, v_{c}\right)-u\left(T_{\beta}, v_{\beta}\right)\right)+ \\
& p_{1}\left(v_{c}-v_{\beta}\right)=36,944.3 \mathrm{~J} / \mathrm{mol} .
\end{aligned}
$$

Our system requires thus a quantity of heat equal to $z \Delta Q$, obtained directly from the laser heating, which we shall now estimate from the time-power record, Fig. 2. We have previously identified in Table 1 the coordinates of states $\beta$ and $c$ in Fig. 2 . The transmitted power varies from 0.7582 to 1.440 $\mathrm{mW}$, with an approximate mean value of $(0.76+$ $1.44) / 2=1.1$. The mean absorbed power, in the time interval from 93.40 to $96.42 \mathrm{~ms}$ is $(36.43-1.1) \mathrm{mW}$ $=35.3 \mathrm{~mW}$, so that the absorbed energy is 35.3 $\mathrm{mW} \times 3.02 \mathrm{~ms}=0.1066 \mathrm{~mJ}$. It follows that $z=$ $2.886 \mathrm{n}$ mol. The system initial volume is $V_{\Sigma, 1}=z v_{1}$ $=1.703 \times 10^{-13} \mathrm{~m}^{3}$. It follows that $\mathrm{pr}^{2}=z V_{\Sigma, 1}=$ $8.514 \times 10^{-10} \mathrm{~m}^{2}$ and $r_{0}=16.46 \mu \mathrm{m}$.

We can also figure out that the recorded power signal Fig. 2 has been obtained when the distance from the system focal point to the chamber middle plane is $2 \mathrm{~mm}$, since it includes nine cycles in a time interval of $117 \mathrm{~ms}$, with a mean period of 13 ms, corresponding to a frequency of $76.9 \mathrm{~Hz}$. According to Fig. 9, there are only two points with a frequency close to 76.9 , those corresponding with $\psi= \pm 2 \mathrm{~mm}$.

\subsection{Private communication with $\mathbf{R}$. Chiu}

The laser beam impinging on the left lens has a radius of $0.4 \mathrm{~mm}$. Thus, it is focused under an angle $\tan \alpha=0.4 / 50=8 \times 10^{-3}$. Since the ratio of the system radius to two millimeters must be tan $\alpha$, the system radius must be $r_{0}=16 \mu \mathrm{m}$. This figure must be compared against our previous result, i.e., 16.46 $\mathrm{mm}$.

Also, $4 \mathrm{~g}$ of Jamaica anthocyanins have been dissolved in $60 \mathrm{~cm}^{3}$. Since the anthocyanins have a mean molar mass of approximately $200 \mathrm{~g}$, we can calculate that $q_{j} \approx 0.04$. Using the obtained value for $\sigma q j$, we obtain for $\sigma=3.148 \times 10^{-23} \mathrm{~m}^{3}$.

\section{CONCLUSIONS}

This work proposes a thermodynamic cycle to understand the experimental data of Chiu et al. [9] which has been adapted from Landau and Lifschitz [10] theory of stable; metastable, those states found between the outer and inner bells in the $P V$ diagram; but especially, those which we call unstable, here represented by the role played by state $c$. To accomplish this project, we had to modify the van der Waals equation of state which depends on two parameters linked with the critical state. Our modified equation of state depends on four, the previous ones, and two more that we had to calculate from room temperature data. The thermodynamic transformations induced by the laser heating give rise to a cycle: $\beta$, c, sudden evaporation, $\phi$, and back to $\beta$ by means of cooling by contact with the chamber walls.

We have given a simple explanation of the pulse frequency observed and also of the pulse width as functions of the distance between the chamber's middle plane and the system focus which depends on only two assumptions:

That the cooling time should depend essentially in the thermodynamic state $\phi$ and the room temperature $T_{1}$, increasing only as the volume of the activation cylinder grows (large $\psi$ ). The heating time depends only in states $\beta$ and $c$, and should be proportional to the volume of the activation cylinder.

${ }^{7}$ There are two points in Fig. 9 which have not been included since they represent simultaneously pulse repetition rate and pulse width, although they fall in the fitted curve. 
It should be remarked that the thermodynamic properties of doped ethanol are well described by those of the pure ethanol, possibly due to a very low concentration $q_{j} \approx 0.04$. Also, the $\rho$ introduced in Fig. 10 should be 0 , that is, the classical approximation is good.

\section{REFERENCES}

[1] M. Graetzel // Applied physics 421 (2003) 586.

[2] C. Qin and A. Clark // Chem. Phys. Lett. 438 (2007) 26.

[3] D. Strack and W. S. T. Vogt // Phytochemistry 62 (2003) 247.

[4] K. Bouchouit, B. Derkowska,

A. Migalska-Zalas, S. Abed, N. Benali-cherif and B. Sahraoui // Carotenoids, Dyes and Pigments 86 (2010) 161.

[5] J. Ramirez, D. Mendoza and V. Castano // Radiat. Meas. 29 (1998) 195.
[6] D. Rangel, J.Navarro, S. Vargas, M.Gonzalez, V.M. Castano and R. Rodriguez // Int. J. Energy Res., doi:10.1002/er.3700.

[7] R. Singer and E. Carreira // J. Am. Chem. 117 (1995) 12360.

[8] M. Reets, M. Drewes and A. Schmitz // Angewandte Chem, doi:10.1002/ anie.198711411.

[9] R. Chiu, M. Mora-Gonzalez, E. VillafanaRauda, F. Casillas-Rodriguez, J. CastanedaContreras, V. Maranon-Ruiz and V. M. Castano // Optik 134 (2017) 216.

[10] L. Landau and E. M. Lifshitz, Course of Theoretical Physics: Statistical Physics, Vol. 5 (Pergamon Press, Oxford, 1980).

[11] Van der Waals constants, https:// en.wikipedia.org/wiki/Ethanol, accessed: 2017-12-01.

[12] J. A. Schroeder, S. G. Penoncello and J. S. Schroeder // J. Phys. Chem. Ref. Data 43, doi:505 10.1063/1.4895394. 